\title{
Letter to the editor regarding an autosomal recessive mutation in SCL24A4 causing enamel hypoplasia in Samoyed and its relationship to breed-wide genetic diversity
}

\author{
Frank W. Nicholas ${ }^{1}$, Cathryn Mellersh ${ }^{2}$ and Tom Lewis ${ }^{3^{*}}$
}

To the editors,

We are writing in relation to the article 'An autosomal recessive mutation in SCL24A4 causing enamel hypoplasia in Samoyed and its relationship to breed-wide genetic diversity' published in Canine Genetics and Epidemiology on 22nd November 2017. Our chief concerns relate to the methodology, namely that: 1 ) using a small number (33) of microsatellite markers that cover only $66 \%(25 / 38)$ of autosomes is suboptimal for determining genetic variation occurring across the genome; and more seriously 2) the sample of dogs from which "breed-wide genetic diversity" was estimated contained a substantial but undisclosed number of close relatives (and so was not a random sample nor representative of the wider breed), meaning that findings pertaining to genetic variation cannot safely be extrapolated without qualification.

The study used microsatellite markers to estimate the genetic diversity of the Samoyed breed. While microsatellites have previously been used to this end, they have largely been superseded by dense SNP chips, where the quantity of bi-allelic marker data provides more precise information on the extent of genetic variation via homozygosity. The study used 33 short tandem repeats (STRs) on 25 autosomes, which is an average of 1.32 STRs per autosome included. However, since Canis familiaris has 38 autosomes, there was no means in this study to determine genetic variation across $1 / 3$ of the genome. It is highly likely that there is genetic variation present within the Samoyed breed on these 13 autosomes, but this study is simply unable to detect its extent. Furthermore, genetic variation at genomic regions on the 25 included autosomes not in linkage disequilibrium with the STRs also cannot be detected. Thus, while this

\footnotetext{
* Correspondence: tom.lewis@thekennelclub.org.uk

${ }^{3}$ Genetics Research Manager, The Kennel Club, Clarges Street, London W1J $8 \mathrm{AB}, \mathrm{UK}$

Full list of author information is available at the end of the article
}

number of STRs may be sufficient for their stated purpose (parentage verification and forensic testing) and an acceptable 'broad brush' means of estimating diversity in a population where there is no alternative (such as wild populations), they are unable adequately to describe the extent of genetic variation across the canine genome, including regions of depleted variation due to selection. Also, it is not immediately evident how the cited website (endnote 14) justifies the claim that "this population [of 182 animals] would identify over 95\% of existing genetic diversity and heterogeneity in Samoyed based on experience with other breeds".

There is also an important issue regarding sampling of the 182 animals in relation to the inference of genetic variation at the 33 STRs in the wider, global breed. Little detail is given regarding the sampling and the likely bias therein. The authors state that "Samples were solicited through web communications and owners/breeders wishing to submit DNA for testing" and report that the sample comprised 144 dogs from North America, 32 from Europe and 6 from Australia (79.1\%, 17.6\% and 3 . $3 \%$, respectively). The preponderance of North American dogs in the sample could imply the sample largely consisted of a (partially inter-related) subsection of the wider breed. Furthermore, given the method of sampling it is conceivable, or likely even, that repeat customers may have resulted in several individual breeders submitting samples from multiple, closely related dogs in their kennels or lines. Indeed, the authors state in the results section that "twenty of 168 (12\%) healthy dogs were found to be heterologous [sic] for the mutation and most were parents or known close relatives of affected dogs", thereby revealing that a significant section of the sample did indeed consist of close relatives, which would have an impact on detected genetic variation. However, other than reporting in the abstract that "This 
population was biased towards close relatives" as a caveat when estimating the population-wide incidence of enamel hypoplasia, the authors provide no further information, such as the degree of relationship among dogs within the unaffected or affected cohorts, which were nevertheless used to determine breed-wide genetic diversity. The authors report that they sought pedigree data and information on relationship to affected dogs, yet provide no evidence of the degree of relationship, as determined by pedigree, of this sample. This is critical to interpretation of the results; the interpretation would be very different if all 182 dogs were part of a large extended family compared to if they were unrelated [as determined from pedigree records] at 5 generations. The authors may point to their internal relatedness (IR) results, with one quarter of Samoyed IR scores between 0.132 and 0.502 putatively indicating a significant degree of parental relatedness, but there is no way of knowing the extent to which this level of relatedness is due to the sampling, as distinct from a reflection of the whole breed. In the conclusion, the authors state that "Samoyed have a lower level of genetic diversity than estimated from prior pedigree or SNP-based studies", but this finding is to be expected given the inability to detect genome-wide variation in the methods employed and a sample that, by the authors' own admission, contains a number of closely-related dogs.

The authors advise in the paper that "the mutation [for SCL24A4] could be safely eliminated without affecting existing genetic diversity". Leaving aside the question of whether this paper provides a realistic estimate of genetic diversity, we would caution against the wholesale exclusion of carriers for breeding for two reasons. Firstly, with the availability of a DNA test for the mutation, breeders have the means to ensure that no affected puppy will ever be produced. So long as the mutation is not under indirect positive selection, the mutation frequency in this scenario is expected to remain static (apart from the chance of new versions of the same variant arising by mutation, which is exceedingly low). Therefore, it is a sensible course of action to make use of the available DNA tests to retain heterozygotes (carriers) in the breeding pool, at least for the first few generations after the DNA test becomes available, thereby decreasing the possibility of an artificially created genetic bottleneck which may lead to a rapid loss of genetic diversity. Selection against the mutation, by using carriers in a decreasing proportion of matings over the course of a number of generations, may still be applied and would result in a gradual reduction in the mutation frequency. Secondly, there are a number of independent selection objectives (even considering only those pertinent to health) in the Samoyed and most other breeds. There are several DNA tests commercially available to Samoyed breeders, including for degenerative myelopathy, hereditary nephritis, X-linked progressive retinal atrophy and oculo-skeletal dysplasia, as well as recommendations to participate in hip dysplasia screening. Because these objectives are most likely independent in genetic aetiology, identifying suitable breeding animals by excluding all those known to be carriers of any of the identified mutations influencing these disorders and that fall below a particular threshold for hip dysplasia would result in a cumulative effect, leaving a considerably smaller pool of breeding candidates and thus potentially creating a genetic bottleneck. Indeed, with identification of an ever-increasing number of mutations that are either the direct cause of, or are influential on the development of, disease, breeders are being faced with an increasing number of objective traits relating to diseases. Therefore, the focus of future breeding strategies will have to be on compensatory pairings of candidates, since very few individuals will have a clean bill of health for all objectives.

\section{Authors' response}

Niels C. Pedersen, Bonnie Shope and Hongwei Liu

Professors Nicholas and Mellersh and Dr. Lewis were concerned about the methodology that led us to conclude that Samoyed lack genetic diversity. They then used these concerns to frame a personal opinion meant to be reassuring to breeders. In doing so, they ignored the major theme of our study, which was to describe a heretofore unrecognized autosomal recessive disorder in Samoyed and the historic and genetic context in which it arose. As veterinarians, we were deeply concerned about identifying health problems of a genetic nature and providing tests to deal with them. We also wanted to make the study a teaching as well as research experience for all breeders.
Their first criticism involved the use of 33 microsatellite markers across only 25/38 autosomes. This was linked to another question pertaining to why microsatellites were even being used at all, given modern SNP technology. The microsatellites chosen for this study were vigorously selected for their ability to discern genetic differences by the International Society for Animal Genetics, with addition of several new markers from our Veterinary Genetics Laboratory. The microsatellites are known to be highly polymorphic and have been used successfully for many studies concerning parentage and genetic diversity. Some of the added markers were for 
the DLA class I and II regions, which contains genes concerned with immune responses. This region is in strong linkage disequilibrium, with one copy inherited by descent from each parent. It is extremely difficult to define the DLA region using SNPs due to the numerous polymorphisms, but it is very efficient and accurate to track this region using microsatellites. DLA haplotypes are powerful identifiers of breed founders. We were also concerned about the fact that our markers did not cover every chromosome and added 25 more microsatellites to cover missing chromosomes. We found that the additional microsatellites, although carefully selected, did not add anything to the power of the current 33 markers to study breed-wide genetic diversity and relatedness. We also compared genetic diversity in Standard Poodles by both microsatellites and 170K SNPs and found no major differences. This same microsatellite panel has been used to successfully study genetic diversity in a number of dog breeds and this information can be found on the Veterinary Genetics Laboratory website https://www.vgl.ucdavis.edu/services/dog.php. We are confident, therefore, that the genetic diversity we were measuring in Samoyed was accurate as described and that employing large panels of SNPs would not significantly alter the conclusions.

The numbers, relatedness, and geographic distribution of Samoyed was questioned, with the implication that we underestimated the genetic diversity in Samoyed compared to earlier pedigree and SNP studies. Although we failed to mention it, more than one hundred of the DNA samples were from Samoyed from around the world submitted to the VGL for other genetic testing and not for the ARAI mutation. One hundred eightyfour Samoyed from around the world is also not a small sampling, at least compared to virtually all other breed studies one might find in the literature. The fact that the 184 Samoyed were genetically similar across the world adds also indicates that most of the existing genetic diversity in the breed was represented in this population.

The reviewers cited other studies involving pedigrees and SNPs as evidence that we may have under-estimated genetic diversity in Samoyed. They failed to mention that the conclusions of these studies were the same. The pedigree study of Bell concluded that the breed had a narrow base of founders and that deep pedigrees showed extensive sharing of the same individuals. A pedigree study conducted by the UK Kennel Club estimated that the effective population size for UK Samoyed was 64.7 dogs. Both studies agreed with our conclusion that genetic diversity in the breed was not great, although adequate. The SNP study quoted by Sorsa used the MyDogDNA array containing around 6788 SNPs, but the study only involved 50 Samoyed and the author admitted that the number was too small to make any conclusion other than that diversity appeared adequate. We also concluded that genetic diversity in Samoyed was derived from a small founder population, but that it was adequate. We have limited faith in pedigrees as sole determinants of genetic diversity.

The second half of the comments pertained to what should be done when a new genetic disorder is identified in a breed and a test for that mutation provided. We did offer three alternative solutions to breeders regarding autosomal recessive enamel hypoplasia: 1) ignore it, 2) eliminate it, or 3) breed around it. We believe that the professors and Dr. Lewis were saying the same things, although they offer a fourth option, i.e., to quickly reduce the incidence of the mutation by testing to an "acceptable" level and live with it. We described the reasons why each of our three approaches might be selected. If the defect is at very low incidence, the genetic diversity sufficient, and breeders are good about random mate selection, ignoring the mutation is a viable option. The chances of getting affected dogs would be extremely small. However, if there is adequate genetic diversity and the incidence of the defect is low, it might be a good idea to eliminate the trait on the perchance that it might be amplified later by some sort of artificial genetic selection (e.g., popular sire effect). We provided our best possible scientific evidence that eliminating the mutation in Samoyed could be accomplished without loss of genetic diversity. We do agree with the reviewers that eliminating genetic traits can lead to serious inbreeding in situations where the incidence of the mutation is high and breedwide genetic diversity low. Such a situation arose for pug dog encephalitis. We identified a region in the DLA that identified carriers of the trait, but when we looked at the incidence of the causative region, over one-third of individuals in the breed were carriers and genetic diversity for the breed was low. Eliminating carriers would seriously reduce existing genetic diversity. Therefore, a recommendation was given to select around the trait by careful mate selection. Fortunately, the trait was recessive, so breeders could select for Normal/Normal or Normal/Carrier puppies. We stand by our conclusion that the ARAI mutation can be safely eliminated from the breed.

We agree that the reviewer's description of the problems that breeders face when having to select against multiple simple and complex genetic traits when given a small number of available dogs, low genetic diversity and geographic limitations. However, the professor's and Dr. Lewis' major conclusion that "the focus of future breeding strategies will have to be on compensatory pairings of candidates, since very few individuals will have a clean bill of health for all objectives" is a bit defeatist. It is also dismissive of the research that went into our study and an admission that all breeds are in trouble and the best that can be done is to choose the 
least objectionable pairings. Since this is precisely what good breeders are already doing, their suggestion is another reassurance from certain geneticists to breeders that "all is right, and nothing needs to change." We have hopefully shown Samoyed breeders that they have not yet reached a point of great concern but forewarned them that Samoyed around the world come from a limited genetic foundation that needs to be carefully managed. The emergence of ARAI in the breed points to the problems facing many other breeds with small numbers and limited genetic diversity. The fact that Samoyed breeders have retained reasonable health through their breed's existence is a plus.

Authors' contributions

All authors drafted, read and approved the final manuscript.

\section{Competing interests}

$\mathrm{TL}$ is a full-time employee of the Kennel Club. FN and CM declare no competing interests.

\section{Publisher's Note}

Springer Nature remains neutral with regard to jurisdictional claims in published maps and institutional affiliations.

\section{Author details}

${ }^{1}$ Emeritus Professor of Animal Genetics, Sydney School of Veterinary Science, University of Sydney, Sydney, NSW 2006, Australia. ${ }^{2}$ Head of Canine Genetics, Animal Health Trust, Lanwades Park, Newmarket CB8 7UU, UK. ${ }^{3}$ Genetics

Research Manager, The Kennel Club, Clarges Street, London W1J 8AB, UK.

Received: 2 March 2018 Accepted: 10 March 2018

Published online: 01 May 2018

Submit your next manuscript to BioMed Central and we will help you at every step:

- We accept pre-submission inquiries

- Our selector tool helps you to find the most relevant journal

- We provide round the clock customer support

- Convenient online submission

- Thorough peer review

- Inclusion in PubMed and all major indexing services

- Maximum visibility for your research

Submit your manuscript at www.biomedcentral.com/submit 